\title{
Hidden Ferronematic Order in Underdoped Cuprates
}

\author{
G. Seibold,${ }^{1}$ M. Capati, ${ }^{2}$ C. Di Castro, ${ }^{2}$ M. Grilli, ${ }^{2}$ and J. Lorenzana ${ }^{2}$ \\ ${ }^{1}$ Institut für Physik, BTU Cottbus, PBox 101344, 03013 Cottbus, Germany \\ ${ }^{2}$ ISC-CNR, CNISM and Dipartimento di Fisica, Università di Roma "La Sapienza", P.le Aldo Moro 5, I-00185 Roma, Italy
}

(Dated: September 22, 2018)

\begin{abstract}
We study a model for low doped cuprates where holes aggregate into oriented stripe segments which have a vortex and an antivortex fixed to the extremes. We argue that due to the interaction between segments a state with macroscopic polarization is stabilized, which we call a ferronematic. This state can be characterized as a charge nematic which, due to the net polarization, breaks inversion symmetry and also exhibits an incommensurate spin modulation. Our calculation can reproduce the doping dependent spin structure factor of lanthanum cuprates in excellent agreement with experiment and allows to rationalize experiments in which the incommensurability has an order parameter-like temperature dependence.
\end{abstract}

PACS numbers: 74.25.Ha, 71.28.+d, 75.25.-j

The question whether there is a broken symmetry hidden order in the pseudogap phase of cuprate hightemperature superconductors is still a matter of debate. In this regard, a popular proposal is unidirectional charge 1, 2] or spin and charge order [3, 4] known as stripes.

Bulk evidence for long-range charge stripe order has only been seen in few compounds. [5 7] $\mathrm{La}_{2-x} \mathrm{Sr}_{x} \mathrm{CuO}_{4}$ (LSCO) shows low energy incommensurate spin scattering, usually associated with some kind of stripe correlation, which undergoes a rotation towards the diagonal direction below hole concentrations $x \lesssim 0.05$, where it even becomes elastic. 8 10 The incommensurability $\delta$ (proportional to the inverse of the spin modulation) evolves linearly $\delta \sim x$ from $x=0.02$ up to the metallic phase where it saturates above $x \gtrsim 1 / 8[11$ as expected for stripes. [12]

Quasistatic incommensurate spin scattering along the $\mathrm{Cu}-\mathrm{O}$ bond direction has also been found in detwinned YBCO. [14, 15] In this case, the absence of signatures of long-range charge order and the evidence of rotational symmetry breaking [14 16] points towards a nematic order. It has been proposed that this order arises either from incipient unidirectional fluctuating stripes [17 or from an independent $\mathrm{d}$-wave type nematic actor which preserves translational symmetry. [18] Other proposals for a broken symmetry state include orbital currents which break time reversal symmetry [19], spirals 20] and a d-density wave. 21, 22

In this paper we show that neutron scattering experiments in strongly underdoped cuprates can be understood in terms of a phase which breaks rotational and inversion symmetry. It is formed by oriented stripe segments which do not need to have positional order thus we call it ferronematic. The segments are oriented because they sustain a vortex and an antivortex of the antiferromagnetic (AF) order in the extremes (Fig. 11). Although formally the phase has zero ordering wave vector in the charge sector, we show that it induces magnetic

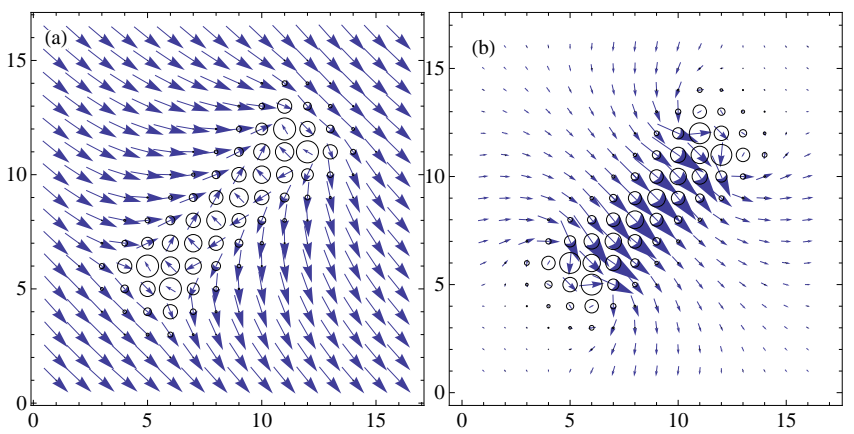

FIG. 1: (color online). a) Stripe segment for 8 holes (corresponding to $4 \mathrm{VA}$ pairs) obtained by minimizing the GA energy of on a $16 \times 16$ lattice in the one band Hubbard models with $t^{\prime} / t=-0.2$. The radius of the circles are proportional to the added hole density while the arrows are the staggered magnetization. b) Spin currents defined from the conservation of the $z$ component of the magnetization 24]. In the continuum limit the spin current is proportional to the gradient of the phase of the staggered magnetization.

incommensurate peaks in excellent agreement with experiment (Fig. 4). Remarkably, the order parameter is proportional to the incommensurability as suggested by experiment. 14, 15]

We focus on non-superconducting underdoped LSCO which can be grown in a structure with only two twin domains with different population. Therefore similarly to the case of YBCO the one-dimensionality of the low energy (diagonal) spin response can be clearly resolved. [9, 10]

Previous variational computations 23 26] for the (low) doping induced spin structure in cuprates suggest the formation of magnetic vortex-antivortex (VA) pairs the relevance of which has been discussed e.g. in context of the destruction of long-range Néel order. [27] In order to study these textures we have performed variational calculations based on the Gutzwiller approximation (GA) of the extended one-band Hubbard model. The ratio be- 
tween on-site repulsion $U$ and nearest-neighbor hopping $t$ is set to $U / t=8$ as suggested by previous studies. 28, 29] The ratio between next-nearest neigbor hopping $t^{\prime}$ and $t$ is taken as a material parameter. 30]

Within this framework the energetically most stable solution for two holes is a VA pair. 24] Our GA computations reveal that the interaction between the pairs is dominated by an anisotropic short-range core-core contribution which originates from the distribution of the localized holes. This results in a head-to-tail aggregation of VA pairs which tend to form chains in such a way that only the vortex and the antivortex on the extremes contribute to the long range distortion. Fig. 11(a) shows the spin and charge structure for 8 holes corresponding to $4 \mathrm{VA}$ pairs. Examination of the spin current (b) allows to visualize the VA pair nucleated at the extremes and the fact that the texture breaks inversion symmetry. Notice that the segments tend to form an antiphase domain wall of the AF order although the transition from finite segments to infinite stripes is non trivial and will be discussed elsewhere. 32.

Fig. 2 shows that segments formed by $2 N$ holes have systematically lower energy than $2 N$ spin polarons with the preferred orientation depending on parameters as discussed below. Seen the segment as formed by $N$ VA pairs we obtain the binding energy $E_{\text {bind }}$ between the pairs from $E(N)=N * E_{1}+(N-1) E_{b i n d}$. Parameters appropriate for lanthanum cuprates $\left(t^{\prime} / t \sim-0.15 \ldots-0.2\right)$ [30], yield a slight preference for diagonally oriented segments. In addition we have checked that in the low temperature orthorhombic (LTO) phase an anisotropy of $t^{\prime} / t$ along orthorhombic a- and b-axis favors the orientation of the VA-pairs along the a-axis.

Until now we have neglected the long-range part of the Coulomb interaction which limits the infinite aggregation of VA pairs. In the spirit of Ref. [31] we may estimate its effect by considering the segments (each hosting $N_{c}$ charges) embedded in a homogeneously charged background. For large $N_{c}$ the Coulomb energy per charge increases logarithmically with the number of holes. At a fixed doping concentration $x$ the energy per planar $\mathrm{Cu}$ reads,

$$
\frac{E\left(N_{c}\right)}{L^{2}}=x\left[E_{c} \ln \left(N_{c}\right)+\gamma+\frac{1}{N_{c}}\left|E_{\text {bind }}\right|\right]
$$

with $E_{c}=e^{2} /\left(\epsilon_{0} a_{o}\right)$ a charging energy expressed in terms of the orthorhombic lattice constant $a_{o}$, the static dielectric constant $\epsilon_{0}$, and $\gamma=\left(E_{1}+E_{\text {bind }}\right) / 2$. The last term comprises the fact that shorter segments have less binding energy. The energy is minimized by $N_{c}=\left|E_{\text {bind }}\right| / E_{c}$ which leads to short segments of only few lattice constants at infinitesimal doping. Considering a Wigner crystal of segments and screening we find that the length of the segments tends to grow rapidly with doping and may even diverge at a critical doping. 32.

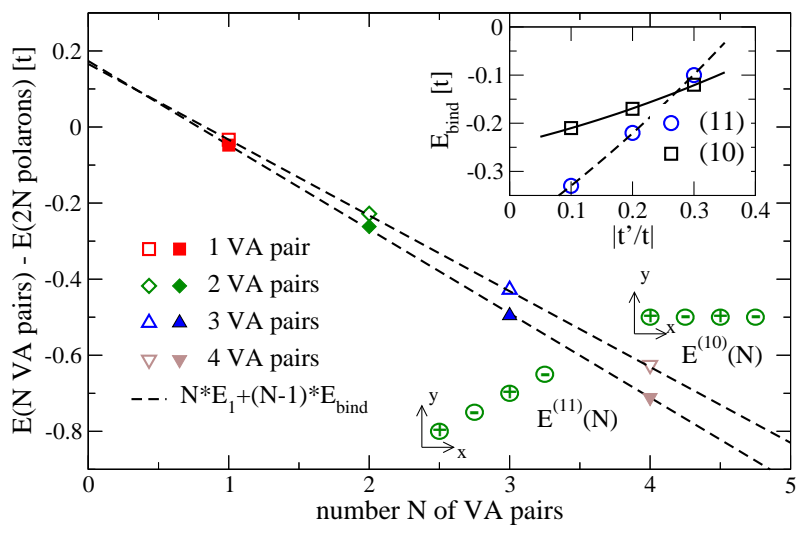

FIG. 2: (color online). Energy of a chain of $2 N$ holes corresponding to $N \mathrm{~V}$-A pairs aligned along the vertical (open symbols) and diagonal direction (full symbols) as compared to the energy of $2 N$ polarons. The slope of the dashed lines corresponds to the binding energy between pairs. Parameters: $U / t=8, t^{\prime} / t=-0.2$. The upper right inset shows the binding energy as a function of $t^{\prime} / t$ for vertical (squares) and diagonal (circles) directions. Lines are guide to eyes. Computations where done in systems with up to $20 \times 20$ sites.

It is useful to remember that a XY vortex can be mapped to a 2D Coulomb charge 37] or alternatively to current wires perpendicular to the $2 \mathrm{D}$ plane. The sign of the charges or the direction of the current is then determined by the vorticity. Thus the VA pairs map into $2 \mathrm{D}$ electric dipoles in the Coulomb analogy or 2D magnetic dipoles perpendicular to the former in the current analogy. In the latter case the magnetic field maps into the spin current which will be used below.

We assume that quenched disorder will yield a state in which segments have no positional order. We have checked numerically 32] that the combination of longrange dipolar interaction plus short range interaction favors a ferronematic alignment of the dipoles.

Information on the spin response of a large aggregate of segments from the GA is hampered by the finite size of the clusters. Since the textures are planar and we are interested in the large scale behavior we consider a classical AF $X Y$-model with nearest neighbor interaction $J$. The segments are modeled as a chain of vacancies which alternately correspond to the center of a vortex and antivortex. The spin structure is then determined from the minimization of the classical energy. In order to prevent annihilation of VA pairs we introduce a frustrating AF second next nearest neighbor coupling $J^{\prime}$ across the segments. The value of $J^{\prime}$ can be obtained by comparing the phase change across a single segment with the GA calculation (Fig. 1) which yields $J^{\prime} \approx J$.

As an additional parameter we have to fix the filling factor $\nu$ which is the number of charges per segment length. Whereas the site-centered VA chain shown in Fig. 11 has $\nu \approx 1$ there exists a similar plaquette centered structure with comparable (though slightly higher) 


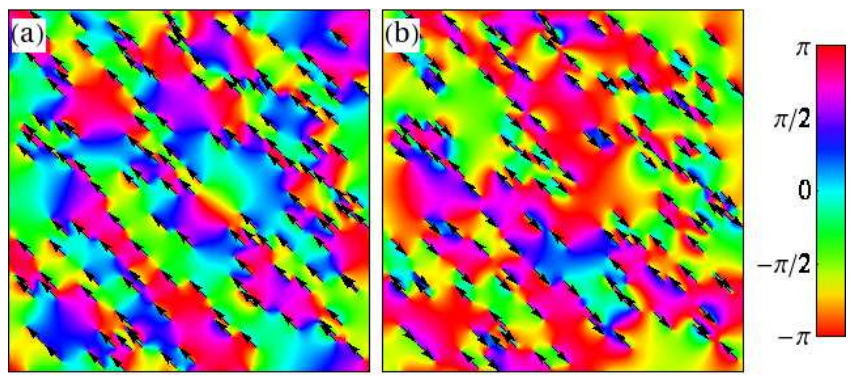

FIG. 3: (color online). Spin phase distribution for (a) macroscopically and (b) randomly polarized distribution of stripe dipoles in a $160 \times 160$ sites system. Segments have a length of 8 sites on the diagonal (4 VA pairs) and are represented by arrows indicating the length and polarization. With a filling factor $\nu=0.7$ the total number of segment sites corresponds to a doping of $x=0.03$.

energy, which has $\nu \approx 0.65$. The filling factor of these structures is thus similar to those of (infinitely extended) diagonal site- and bond-centered stripes. 34] The following calculations assume $\nu=0.7$. Note, however, that experimental data imply an increase towards $\nu=1$ close to the AF boundary. 10]

Fig. 3(a) reports the spin phase distribution for a particular distribution of stripe dipoles with segment length of 8 sites at $x=0.03$, all polarized along the $[-1,1]$ direction. One observes a monotonous increase on the phase of the staggered magnetization along the $[1,1]$ direction, although the distribution of stripe segments is completely random. In Fig. 3(b), for the same distribution of segments, the associated dipole orientation is now completely random. In this case the system disaggregates into large areas with similar phase.

The numerical results can be understood by considering the long-range distortion produced by the segments. Using the magnetic analogy mentioned above and standard arguments from the theory of macroscopic dielectrics and magnetized systems [38] it is easy to see that the system develops a macroscopic "magnetic field" equivalent to an average net spin current perpendicular to the segments orientation and proportional to the average polarization.

Analogously we can obtain this result by considering the influence of a collection of equally oriented segments on spins at point $\mathbf{r}$

$$
\begin{aligned}
& S^{x}(\mathbf{r})=S_{0} \exp (i \mathbf{Q} \cdot \mathbf{r}) \cos \Phi(\mathbf{r}) \\
& S^{y}(\mathbf{r})=S_{0} \exp (i \mathbf{Q} \cdot \mathbf{r}) \sin \Phi(\mathbf{r})
\end{aligned}
$$

where $\mathbf{Q}=(\pi, \pi)$ is the $\mathrm{AF}$ wave-vector and the phase $\Phi(\mathbf{r})$ can be expressed by mapping the Cartesian plane into the complex plane $(x, y) \rightarrow z=x+i y$,

$$
\Phi(z)=\operatorname{Im} \sum_{i=1}^{N_{\text {seg }}}\left[\ln \left(z-z_{i,-}\right)-\ln \left(z-z_{i,+}\right)\right] .
$$

Eqs. (2),(3) yield the angular spin distortion due to the $N_{\text {seg }}$ segments with \pm "charges" at $z_{i, \pm}\left(\mathbf{r}_{i, \pm}\right)$.

By direct integration we obtain that the average spin current in a square system of dimension $L^{2}$ in the direction perpendicular to the "dipole moment" $\mathbf{p}_{i}=\mathbf{r}_{+}-\mathbf{r}_{-}$ is $\langle\nabla \Phi(\mathbf{r})\rangle=\pi \mathbf{p}_{i} \times \hat{z} / L^{2}$ with $\hat{z}$ pointing out of the plane. The macroscopic spin current is given by $\nabla \Phi(\mathbf{r})_{\text {mac }}=$ $\pi \hat{z} \times \mathbf{P}$ where $\mathbf{P}$ is the macroscopic polarization in the charge analogy $\left(\mathbf{P}=\mathbf{p} N_{\text {seg }} / L^{2}\right.$ for identical dipoles $)$. Clearly $\mathbf{P}$ plays the role of the ferronematic order parameter. From Eq. (2) one obtains that the macroscopic spin current implies an incommensurate spin response perpendicular to the segments with $\mathbf{q}=\nabla \Phi(\mathbf{r})_{m a c}$. This is one of our central results and relates the incommensurability with the ferronematic order parameter.

The doping dependence of the order parameter can be estimated by considering segments (number of sites $=l+1$ ) which host $N_{c}$ charges so that the filling factor $\nu=N_{c} /(l+1)$. Since $x=N_{c} N_{\text {seg }} / L^{2}$ is the concentration of charge carriers one thus finds a linear dependence of the incommensurability on doping

$$
q_{\perp}=2 \pi \frac{x}{2 \nu} \frac{l}{l+1} \equiv \pi P .
$$

It is interesting to remark that if one computes the incommensurability of infinite stripes consisting of collinear domain walls (thus without spin current) with filling $\nu$ [3, 12] it coincides with the incommensurability of finite ferronematic segments with the same filling and at the same doping, i.e. such that the total length of the segments coincides with the total length of the stripes. Thus experimentally the two phases are not easily distinguished in the magnetic channel and the main difference will arise in the charge channel with equally spaced stripes producing Bragg peaks in contrast to diffusive scattering in the case of segments.

For a set of configurations of macroscopically polarized VA segments as shown in Fig. 3(a) we now evaluate the magnetic neutron cross section for different dopings which in Fig. 4 is compared with elastic neutron scattering data from Ref. 9. The specific scattering geometry (Fig. 2b of Ref. 9) which is composed of two twin domains with population $2: 1$ has been taken into account. This gives rise to the asymmetry of the spectra since $Q_{A F}$ of the B-twin does not coincide with $Q_{A F}$ of the A-twin. The incommensurate peak position $q_{c}$ is determined by Eq. [4 and is essentially independent from the segment length $l$ (i.e. the dipole moment $p$ ). 39] On the other hand the length influences the peak width as can be seen in the lowest panel of Fig. (4). By decreasing the dipole moment $p$ at fixed doping, the increasing number of segments decreases the fluctuations of the dipole polarization. The strength of the incommensurate response is then favored with respect to the commensurate one. As can be seen from Fig. 4 one finds excellent agreement with the experimental data for segments with 4 VA pairs 


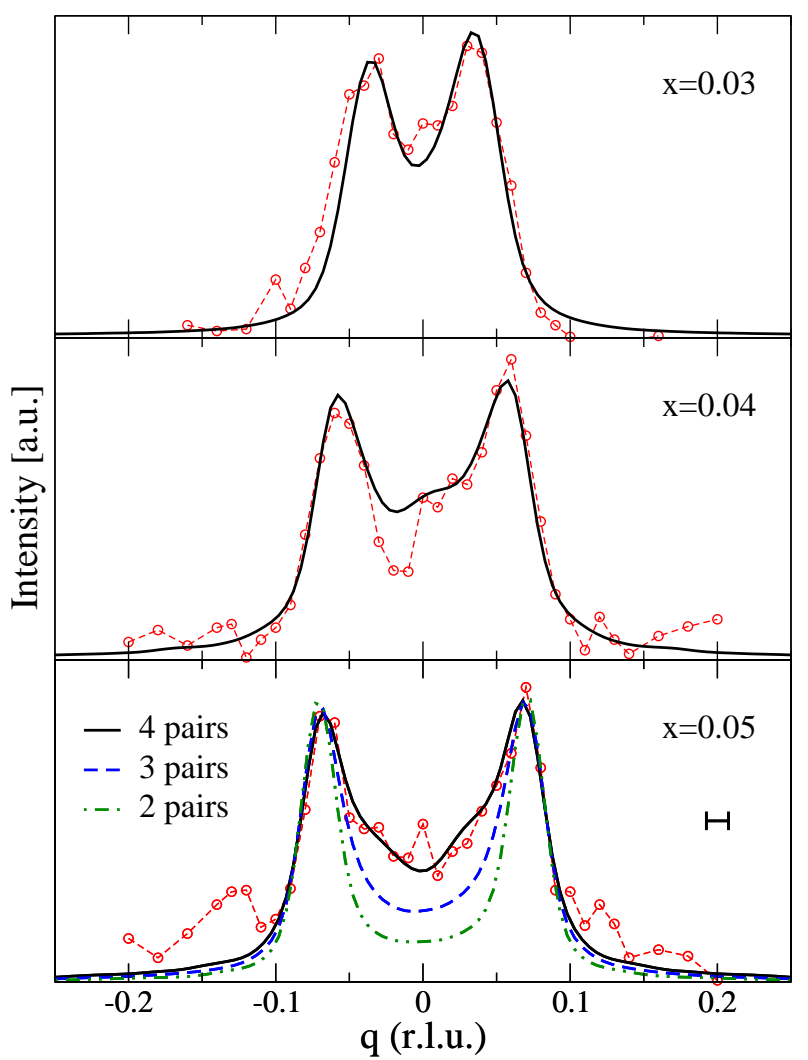

FIG. 4: (color online). Fits of the spin structure factor (LSCO) at different dopings for $N_{c}=8$ segments as explained in the text. For $x=0.05$ we also show spectra for $N_{c}=4,6$ segments for comparison. Computations have been done on lattices with up to $160 \times 160$ sites and we average over $20-30$ segment configurations where the experimental resolution (horizontal bar in the lower panel, cf. Fig. 4 of Ref. 9) has been taken into account by convoluting with a gaussian. Data by courtesy of S. Wakimoto.

(i.e. 8 sites) whereas shorter segments underestimate the intensity at $Q_{A F}$ (cf. lower panel of Fig. (4).

As shown in the inset to Fig. $2\left|E_{b i n d}\right|$ decreases with increasing $\left|t^{\prime} / t\right|$, the reduction is, however, more pronounced for diagonal VA pairs. This results in a crossing of the alignments as a function of $N$ for $-0.3<t^{\prime} / t<$ -0.2 . In addition the lattice structure can influence the orientation. This explains the fact that a diagonal incommensurate spin response has only been observed in nonsuperconducting underdoped lanthanum cuprates where $\left|t^{\prime} / t\right|$ is small [30] and which display a LTO structure in the underdoped regime. At higher doping an increased fraction of local octahedral tilts with LTT symmetry [35] may drive a reorientation of the stripe segments from diagonal to the copper-oxygen direction at the insulator metal transition $x \approx 0.05$. In $\mathrm{Bi}_{2} \mathrm{Sr}_{2} \mathrm{CaCu}_{2} \mathrm{O}_{8+\delta}$ compounds where $t^{\prime} / t$ is large [30] we expect alignment of the segments along the $\mathrm{Cu}-\mathrm{O}$ direction resulting in an inequivalence of hole density on the corresponding $\mathrm{x}$ - and -y oxygen sites. It therefore would be interesting to in- vestigate whether this feature can account for the intraunit cell nematicity observed by scanning tunneling microscopy [36] in these materials.

Apart from the excellent agreement found for the spin structure factor other facts point to the correctness of our interpretation. We expect that thermal fluctuations will disorder the dipole orientation reducing the order parameter until a thermodynamic phase transition occurs. It is not clear whether the high temperature state will be only nematic as in Fig. 3(b) with a second transition at higher temperature to an isotropic state of fully disordered dipoles or if the transition will be directly to the isotropic state. In both cases the incommensurability should display an order parameter behavior. In agreement with this expectation an ordered parameter like temperature dependence of the incommensurability has already been noticed in YBCO. 14, 15]

Since the ferronematic state breaks inversion symmetry one expects on general grounds 40] that it will lead to a real ferroelectric distortion, i.e. to become multiferroic, through e.g. the inverse Dzyaloshinskii-Moriya mechanism. 41] Indeed, a ferroelectric state has recently been detected in strongly underdoped lanthanum cuprates. 33 Unfortunately, a small number of free carriers will make the effect undetectable with capacitance measurements which may explain why it has been seen only at very low temperatures. An appealing possibility would be to look for inversion symmetry breaking with second harmonic generation (SHG) which does not require perfect insulating behavior. [42] We expect that the SHG signal as a function of temperature tracks the incommensurability.

The macroscopic constant spin current can be considered as an average spiral behavior of the spins. In this sense our theory has some similarity with the proposal of Ref. 20]. However, in our case the spiral is a collective effect which is slaved by the ferronematic order of the stripe segments.

Concluding, we have proposed a new phase for strongly underdoped cuprates which breaks $C_{4}$ rotational and inversion symmetry. Our theory provides a consistent explanation for the elastic incommensurate response seen by magnetic neutron scattering experiments and reconciles it with the lacking signatures of charge order. It remains to be seen how the nematic segments act as seeds which lead to smectic correlations (stripes) in some of the cuprate materials.

We thank S. Caprara, R. De Renzi, S. Sanna and P. Carretta for insightful discussions and S. Wakimoto for sending to us the data shown in Fig. 14. J. Lorenzana is supported by Italian Institute of Technology-Seed project NEWDFESCM. G. Seibold acknowledges support from the Deutsche Forschungsgemeinschaft. 
[1] V. J. Emery and S. Kivelson, Physica C 209, 597 (1993)

[2] C. Castellani, C. Di Castro and M .Grilli, Phys. Rev. Lett.75, 4650 (1995).

[3] J. Zaanen and O. Gunnarsson, Phys. Rev. B40, 7391 (1989).

[4] K. Machida, Physica C 158, 192 (1989).

[5] P. Abbamonte, A. Rusydi, S. Smadici, G. D. Gu, G. A. Sawatzky, and D. L. Feng, Nature Phys. 1, 155 (2005).

[6] J. Fink, E. Schierle, E. Weschke, J. Geck, D. Hawthorn, V. Soltwisch, H. Wadati, H.-H. Wu, H. A. Dürr, N. Wizent, B. Büchner, and G. A. Sawatzky, Phys. Rev. B 79, 100502 (2009).

[7] J. M. Tranquada, B. J. Sternlieb, J. D. Axe, Y. Nakamura, and S. Uchida, Nature (London) 375, 561 (1995).

[8] S. Wakimoto, G. Shirane, Y. Endoh, K. Hirota, S. Ueki, K. Yamada, R. J. Birgeneau, M. A. Kastner, Y. S. Lee, P. M. Gehring, and S. H. Lee, Phys. Rev. B 60, R769 (1999).

[9] S. Wakimoto, R. J. Birgeneau, M. A. Kastner, Y. S. Lee, R. Erwin, P. M. Gehring, S. H. Lee, M. Fujita, K. Yamada, Y. Endoh, K. Hirota, and G. Shirane, Phys. Rev. B 61, 3699 (2000).

[10] M. Matsuda, M. Fujita, K. Yamada, R. J. Birgeneau, M. A. Kastner, H. Hiraka, Y. Endoh, S. Wakimoto, and G. Shirane, Phys. Rev. B 62, 9148 (2000).

[11] K. Yamada, C. H. Lee, K. Kurahashi, J. Wada, S. Wakimoto, S. Ueki, H. Kimura, Y. Endoh, S. Hosoya, G. Shirane, R. J. Birgeneau, M. Greven, M. A. Kastner, and Y. J. Kim, Phys. Rev. B 57, 6165 (1998).

[12] J. Lorenzana and G. Seibold, Phys. Rev. Lett. 89, 136401 (2002)

[13] M. Matsuda, M. Fujita, K. Yamada, R. J. Birgeneau, Y. Endoh, and G. Shirane, Phys. Rev. B 65, 134515 (2002).

[14] V. Hinkov, D. Haug, B. Fauqué, P. Bourges, Y. Sidis, A. Ivanov, C. Bernhard, C. T. Lin and B. Keimer, Science 319, 597 (2008)

[15] D. Haug, V. Hinkov, Y. Sidis, P. Bourges, N. B. Christensen, A. Ivanov, T. Keller, C. T. Lin, B. Keimer, New J. of Phys., 12, 105006 (2010).

[16] R. Daou, J. Chang, David LeBoeuf, Olivier CyrChoinière, Francis Laliberté, Nicolas Doiron-Leyraud, B. J. Ramshaw, Ruixing Liang, D. A. Bonn, W. N. Hardy, and Louis Taillefer, Nature 463, 519 (2010).

[17] M. Vojta, Adv. Phys. 58, 699 (2009).

[18] K. Sun, M. J. Lawler, and E.-A. Kim, Phys. Rev. Lett. 104, 106405 (2010).
[19] C. M. Varma, Phys. Rev. Lett. 83, 3538 (1999).

[20] O. P. Sushkov and V. N. Kotov, Phys. Rev. Lett. 94, 097005 (2005).

[21] L. Benfatto, S. Caprara and C. Di Castro, Eur. Phys. J B 1795 (2000).

[22] S. Chakravarty, R. B. Laughlin, D. K. Morr, and C. Nayak, Phys. Rev. B 62, 4880 (2000)

[23] J. A. Vergés, E. Louis, P. S. Lomdahl, F. Guinea, and A. R. Bishop Phys. Rev. B 43, 6099 (1991).

[24] G. Seibold, Phys. Rev. B 58, 15520 (1998).

[25] M. Berciu and S. John, Phys. Rev. B 69, 224515 (2004).

[26] H. Koizumi, J. Phys. Soc. Jpn. 77, 034712 (2008).

[27] C. Timm and K. H. Bennemann, Phys. Rev. Lett. 84, 4994 (2000).

[28] G. Seibold and J. Lorenzana, Phys. Rev. Lett. 94, 107006 (2005).

[29] J. Lorenzana, G. Seibold, R. Coldea, Phys. Rev. B 72, 224511 (2005).

[30] E. Pavarini, I. Dasgupta, T. Saha-Dasgupta, O. Jepsen, and O. K. Andersen, Phys. Rev. Lett. 87, 047003 (2001).

[31] J. Lorenzana, C. Castellani, and C. Di Castro, Europhys. Lett. 57, 704 (2002); Phys. Rev. B 64, 235127 (2001).

[32] G. Seibold et al., unpublished.

[33] Z. Viskadourakis, I. Radulov, A. P. Petrović, S. Mukherjee, B. M. Andersen, G. Jelbert, N.S. Headings, S.M. Hayden, K. Kiefer, S. Landsgesell, D.N. Argyriou, and C. Panagopoulos, arXiv:1111.0050

[34] G. Seibold and J. Lorenzana, Phys. Rev. B 80, 012509 (2009).

[35] E. S. Bozin, S. J. L. Billinge, G. H. Kwei, and H. Takagi, Phys. Rev. B 59, 4445 (1999).

[36] M. J. Lawler, K. Fujita, Jhinhwan Lee, A. R. Schmidt, Y. Kohsaka, Chung Koo Kim, H. Eisaki, S. Uchida, J. C. Davis, J. P. Sethna, and Eun-Ah Kim, Nature 466, 347 (2010).

[37] P. Minnhagen, Rev. Mod. Phys. 59, 1001 (1987).

[38] J.D. Jackson, Classical Electrodynamics, (Wiley, New York, 1998).

[39] Note that Eq. (4) was derived without allowing the background spins to relax. On the other hand the explicit calculations leading to Fig. 4 were carried out by minimizing the magnetic energy. This even more reduces the dependence of $q_{c}$ on $p$.

[40] M. Mostovoy, Phys. Rev. Lett. 96, 067601 (2006).

[41] I. A. Sergienko and E. Dagotto, Phys. Rev. B 73, 094434 (2006

[42] M. Fiebig, J. Phys. D 38 R123 (2005) 